\title{
Using Ghana's 32-m radio telescope to promote astronomy outreach
}

\author{
Alongside its role in astronomical research, the newly commissioned 32-m radio telescope is being used as \\ an outreach tool for astronomy education in Ghana. The observatory also serves as a facility for international \\ postgraduate programmes.
}

\section{R. Aworka, E. Proven-Adzri, T. Ansah-Narh, J. Koranteng-Acquah and E. Aggrey} $\mathrm{n}$ West Africa, and specifically Ghana,
interest in cultural astronomy is more
typical than modern astronomy. In fact,
recent studies on cultural astronomy $y^{1,2}$ have
shown that Africans had ancient knowledge
of the characteristics of people and their
association with the sky. This association
involved physical things such as knowing
the time, the seasonal calendars for farming
activities, and so on, as well as supernatural
beliefs in celestial deities, the influence of
the planets, and astrology. Knowledge of
these activities has helped humans to plan
their lives for generations, especially hunters,
fisherfolk, and farmers.

Meanwhile, modern astronomy research in Ghana is rather undeveloped within our educational settings except for a few course modules such as the DARA basic programme ${ }^{3}$, which aims to provide young Africans with basic skills in radio astronomy, the fundamentals of high-performance computing and big data techniques. On the observational side of astronomy, recently Ghana joined the radio astronomy world when it partnered with South Africa, the United Kingdom, and several other international collaborators to convert the redundant 32-m telecommunications antenna at Kutunse Intelsat Satellite Earth Station (in a suburb of Accra) into a radio telescope (Fig. 1). This makes Ghana the second country in Africa to host a radio telescope and brings a new phase of astronomy into the country.

The expected scientific operations of the new observatory can be classified as follows:

- Single-dish observations. Taking observations as a standalone instrument, including: maser monitoring using spectroscopy, where masers in star-forming regions are monitored to understand their periodic variations; pulsar observations, where pulsars are monitored to measure the glitches and long-term behaviour; radio-continuum flux measurements, where we monitor

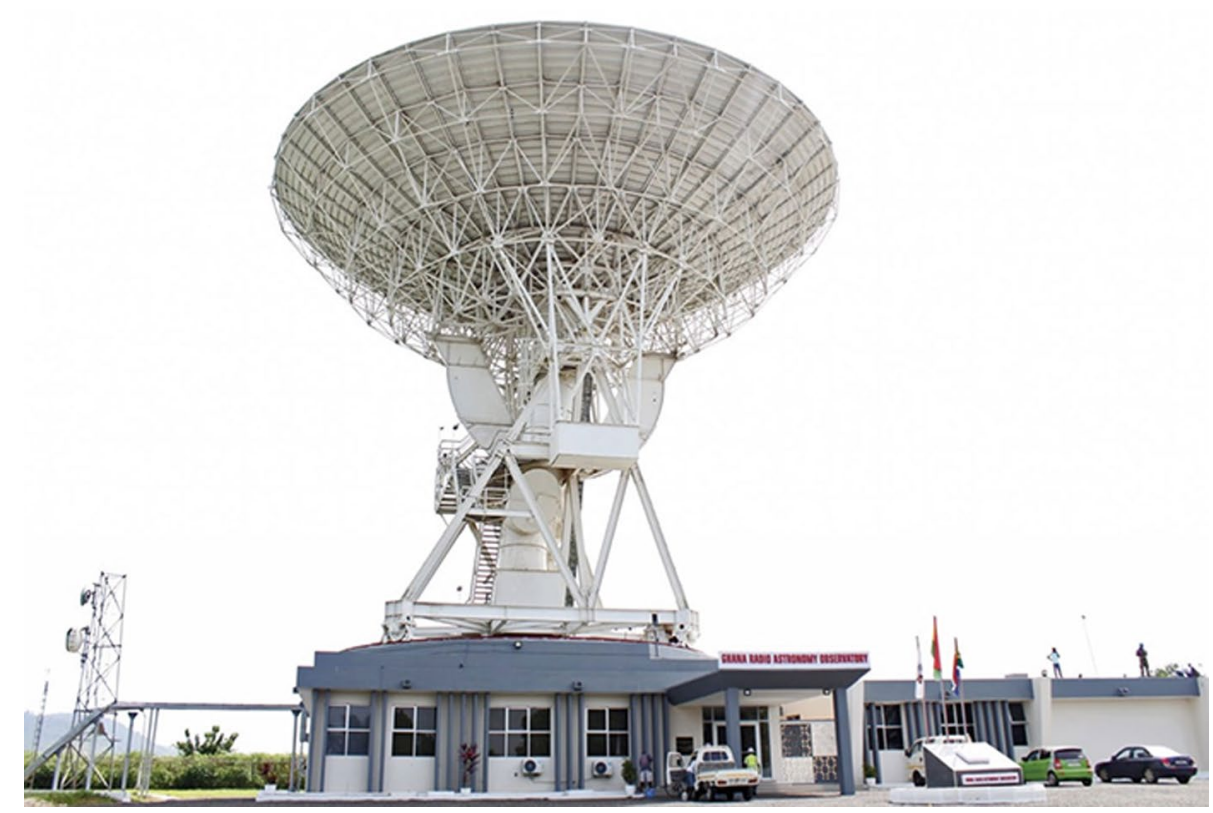

Fig. 1 | The 32-meter radio telescope at the Ghana Radio Astronomy Observatory. The dish was previously used as a satellite Earth station.

radio emissions from gamma-ray flare sources; and wideband multi-channel radiometry, where we perform daily calibration of the receiver using standard radio astronomy calibration sources.

- Very long baseline interferometry (VLBI) observations. Simultaneous observations with other telescopes across the world. The telescope makes up part of the African VLBI Network (AVN) and other global networks such as the Joint Institute for Very Long Baseline Interferometry European Research Infrastructure Consortium.

Although there were launch celebrations for the telescope in Ghana, most of the people in the country do not understand the observatory's benefits. Therefore, the Ghana Radio Astronomy Observatory (GRAO), part of the Ghana Space Science and Technology Institute (GSSTI), has become strongly involved in science outreach and public engagement in order to teach radio astronomy to the public. This Comment aims to describe the approach adopted by us to create an awareness of radio astronomy using the $32-\mathrm{m}$ radio telescope as a focal instrument. We believe this approach will serve as a boost to achieving the United Nations Sustainable Development Goals (SDGs), specifically on targets 4, 5 and 10.

\section{Benefits of astronomy}

Astronomy has been one branch of science that has contributed many amazing industrial applications that have improved the livelihoods of humans and the environment. In a quest to observe and study the universe, the instrumentation, 

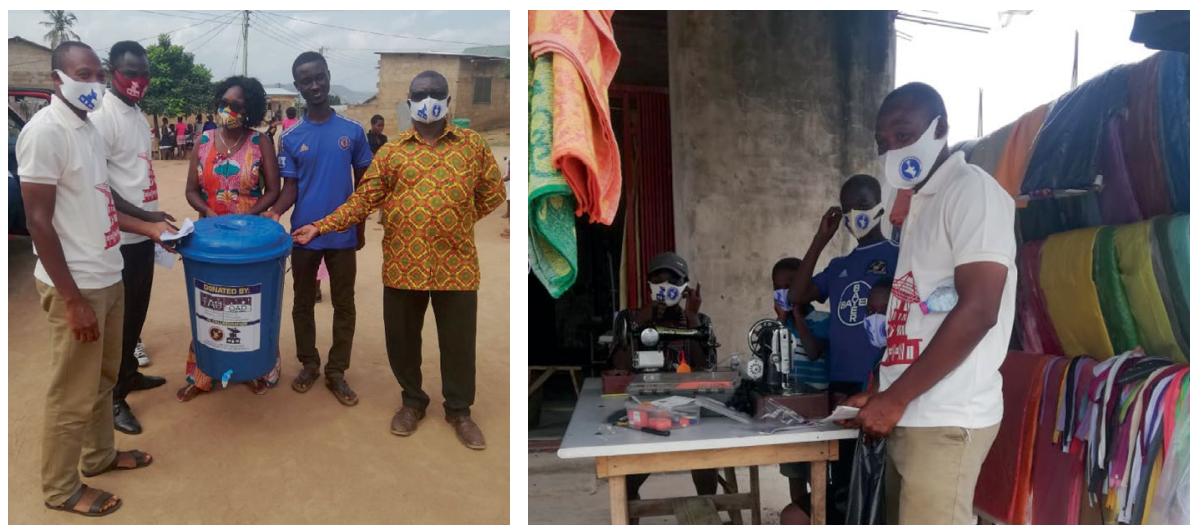

Fig. 2 | Astronomers helping during the COVID-19 outbreak. A GRAO and PRAGSAC team distributing PPE and educating the community about severe acute respiratory syndrome coronavirus 2 (COVID-19) disease.

software and skills developed by engineers and scientists have much potential to spin off into many great applications outside the domain of astronomy. In this section, we discuss some benefits of astronomy to society and particularly, focus on the benefits of GRAO.

Ecological applications. In Ghana, forests and bodies of water are being depleted at an alarming rate, because of illegal mining (popularly known as galamsey). In order to fight against illegal mining activities, Ghana has resorted to using satellite data provided by NASA to help track these illegal acts. When an ecosystem is disturbed or destroyed, some species deplete, whereas others are exterminated. Ecologists have preserved endangered species by resorting to measures that can help monitor and protect these species. For instance, a detection technique using drones and infrared cameras has been used to monitor endangered species.

Innovative research and human capacity building. The GSSTI has been involved in research activities that are geared towards addressing the UN SDGs. The research activities include, but are not limited to, the use of remote-sensing for climate information studies such as drought indicators, pollution monitoring, crop-yield estimation, and agricultural evapotranspiration mapping. In addition, the Institute has been mapping the conversion of vegetation to artisanal gold mining sites. At the same time as the Radio Astronomy and Astrophysics Centre of the Institute has been conducting astronomical research, it has also been behind spin-off research works using big data techniques. Some of the big data projects are using deep learning pipelines to detect the following: pneumonia in chest X-rays images, corn leaf diseases from images, anomalies in electrocardiograms, and COVID-19 using CT scans or X-ray images.

GRAO contribution. Besides the contribution to astronomical research ${ }^{4,5}$, GRAO is also building upon human capacity and improving the socio-economic status of the immediate community and the country as a whole. As part of human development, local electrical and mechanical engineers are being trained by AVN experts to complete the second phase of the dish upgrade.
Furthermore, technicians are being trained to operate the telescope as well as perform regular maintenance. The DARA project is also training and supporting young indigenous scientists in radio astronomy and data reduction, with more than 50 young Ghanaians having benefitted from this programme.

Furthermore, the observatory serves as a training centre for students from the other AVN partner countries (Namibia, Botswana, Zambia, Madagascar, Mauritius, Mozambique and Kenya). For instance, in 2017, the West African International Summer School for Young Astronomers (WAISSYA) was held in Ghana and included some sessions at the observatory. In terms of socio-economic impact, the roads leading to the observatory have been constructed, bringing relief to motorists in the community and opening up economic activities. The hospitality industry in the immediate vicinity also gets a huge boost as scientists, students and visitors come to the observatory. During the COVID-19 outbreak, the observatory, in partnership with the Promoting Radio Astronomy in Ghana through School visits and Astronomy Clubs (PRAGSAC), offered a helping hand to the Kutunse community by sharing personal protective equipment (PPE) (Fig. 2) and also with the broadcasting of preventive measures to take during this period. Note that this COVID-19 awareness project was supported by the International Astronomical Union's Office of Astronomy for


Fig. 3 | Antenna tour. Snapshots of students and facilitators touring the Ghana Radio Astronomy Observatory at Kutunse. 


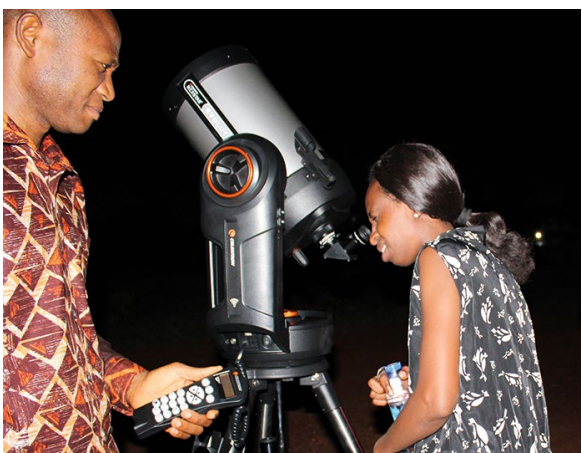

Fig. 4 | Optical telescope observation. DARA students using the optical telescope to observe visible sources in the sky.

Development. We also took the opportunity to educate the community about the benefits of radio astronomy as well as the challenge GRAO faces with human encroachment.

Another important benefit to the country is the introduction of a hydrogen maser - a precision reference clock - to the observatory, which will also serve as a standard reference time for the whole country. Finally, GRAO and PRAGSAC members have developed online lessons in basic astronomy, the Python programming language, and careers in astronomy for high-school students (see https://www.youtube.com/ results?search_query=Pragsac).

\section{Outreach strategies and targets}

As mentioned earlier, radio astronomy is a subject that is not well known among the Ghanaian public. This makes the transfer of information to the public very challenging. To bridge this gap, scientists at GRAO, GSSTI and volunteers from PRAGSAC engage with various schools, universities, teacher bodies and clubs to educate them in astronomy. Sometimes, schools visit the observatory as part of their educational and industrial excursions. At other times, the astronomy teams also visit some schools as part of GRAO outreach efforts to create awareness. Workshops (like DARA and WAISSYA) and conferences (like Dish Conversion) covering broad topics in astronomy and instrumentation are also held. These are some of the strategies we are taking to make radio astronomy known to the public. In this section, we highlight some of these strategies for outreach and also discuss the future targets of GRAO.

Visits to the observatory. Discoveries about the cosmos are exciting to children. It is often said that "astronomy is the gateway to science and technology" ${ }^{\text {. }}$. This rings true because when kids hear about all the amazing discoveries about the Solar System and newly discovered galaxies, they become highly motivated to delve into scientific fields in the course of their education. Therefore, visits to the observatory to learn about radio astronomy is one outreach plan encouraged by the GRAO team.

The type of the target audience we have determines the communication mode of the outreach, with high-school and university groups taught different concepts to primary-school kids. For example, for these younger children, radio astronomy images are not as attractive as optical images. Therefore, we try to pursue different exciting procedures and content to convey fundamental concepts and involve our audience by connecting to their daily experience and pre-existing knowledge. High-school and university groups normally organize these visits to the radio telescope site as part of their courses pertaining to engineering and science or general physics. Since the audience has a good level of background knowledge, we are able to show them how we obtain data about celestial objects. Equations and technical terms are also explained, which give these students some level of understanding regarding the operations and usefulness of radio telescopes and astronomy. Current research at the observatory such as that involving pulsars, methanol masers, machine learning and high-performance computing applications are also briefly explained to the participants.

After the presentation, the students are taken on an interactive tour around the facility as displayed in Fig. 3. At this point, the students are taken to the radio telescope and are shown the different components and their respective functions, particularly the main dish, sub-reflector, alidade structure, mirrors, beam waveguide, elevation and azimuth platforms, motors, shock absorbers, stow pin, wheel tracks and grabbers, receiver, feed-horn, antenna steering control system and back-end computing systems. A physical description of the 32-m beam waveguide radio telescope with mirror configuration is presented in ref. ${ }^{7}$ using a computer-aided drawing and finite element approach. It is an educational tour that makes students appreciate the science of all these parts and yearn to spend time knowing more. In the case of school children visiting the site, special skills in pedagogy are needed to be able to transfer knowledge to them. At the observatory we combine both videos and pictures in the presentations to get the children excited. In addition, to ensure the kids understand parts of the instrument, we involve them in making a card model of a radio telescope using paper and glue.
Visits to schools. As students visit the observatory, we also visit schools to create awareness in astronomy. Research scientists from the observatory go to tertiary-level schools to lecture on the projects being carried out and also to educate students on the opportunities available in the area of astronomy. The institute, as a way of reaching the audience in the basic schools, initiated a project with the objective of working through school visits and school clubs to give students practical astronomy information and show them the importance and benefits of the GRAO at Kutunse. In fact, the PRAGSAC group focuses on visiting basic schools assisting in the development of learning materials as well as the formation of astronomy clubs.

Workshops and conferences. Organizing training programmes and conferences are further steps towards reaching our audiences. The GSSTI and GRAO have organized a number of workshops and conferences over the years. The initiative is mostly via the collaboration of the UK's Leverhulme-Royal Society Africa Awards and the Newton Fund, which supports the DARA project. Training is being organized to use the telescope as a tool for astronomy education in Ghana. In addition, the dish conversion workshop organized in 2019 and mentioned earlier was the first of its kind in Africa. The purpose of this workshop was to engage public and private-sector stakeholders and experts to broaden awareness and share knowledge in radio astronomy for sustainable development. The workshop received participants from Africa, Latin America, Europe and Asia.

In 2017, the third episode of the WAISSYA workshop was organized to develop interest in astronomy in students and teachers in Ghana and West Africa. During this summer school, participants were divided into two focus groups, undergraduate and postgraduate, where each group had both lectures and hands-on practicals. The PRAGSAC team has also held a number of workshops for teachers in the basic schools to update them on astronomy and new ways to teach the children. Additionally, the DARA programme takes in about twelve qualified students in the field of physics and other related fields per year and gives them basic training courses in radio astronomy: astrophysics, technical training in using the radio telescope for observations, data reduction and analysis. This training is given by experts in radio astronomy from the UK, other parts of 
Europe, India, South Africa, Japan and Ghana. As part of the training, the DARA participants also use an optical telescope for night viewing (Fig. 4).

\section{Future targets and outreach channels.} In order to reach out to a large number of people in the country, the outreach team will make use of social media platforms to catch the attention of parents and philanthropists too. We will also present at science education conferences. GRAO is also exploring funding avenues to build a mobile or fixed planetarium, produce documentaries and fliers to reach a bigger audience.

Efforts are being made to improve gender diversity in astronomy in Ghana and GRAO. The staff count at GRAO is eight, of which $50 \%$ are women. To encourage more girls into the field of astronomy, the female staff mostly facilitate the outreach programmes. Citing another example, for the 2021/2022 DARA training at GRAO, all of the female applicants were selected, resulting in six male and six female participants. The diversity has improved appreciably over the last few years and prospects are looking even better.

\section{Conclusion}

The socio-economic gain from astronomy is not very well recognized in Ghana and so creating awareness among the general public would be an efficient way to promote and communicate GRAO's involvement in science education and capacity building. This Comment has given a brief introduction to the outreach and training activities undertaken at GRAO and also outlined some of the benefits of the observatory to the Ghanaian society.

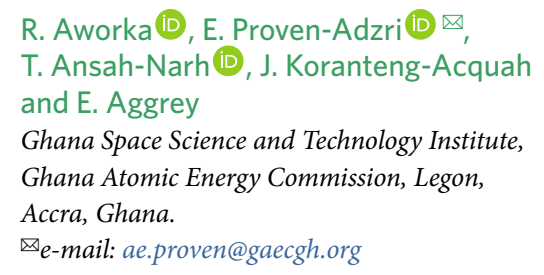

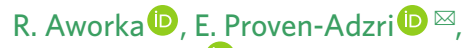
T. Ansah-Narh (D), J. Koranteng-Acquah and E. Aggrey

Ghana Space Science and Technology Institute, Ghana Atomic Energy Commission, Legon, Accra, Ghana.

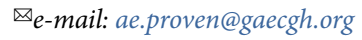

Published online: 13 December 2021 https://doi.org/10.1038/s41550-021-01555-1
References

1. Vannier, C. Anthropol. News 60, e53-e56 (2019).

2. Holbrook, J. J. Astron. Cult. https://escholarship.org/uc/item/ 7jg3m $3 \mathrm{z} 9$ (2016).

3. Hoare, M. G. Nat. Astron. 2, 505-506 (2018).

4. Venter, M. \& Bolli, P. IOP Conf. Ser.: Mater. Sci. Eng. 321, 012003 (2018).

5. Reynard, L. Quest 13, 10-13 (2017).

6. Salimpour, S. et al. Res. Sci. Educ. 51, 975-996 (2021).

7. Azankpo, S. Surface Accuracy and Pointing Error Prediction of a $32 \mathrm{~m}$ Diameter Class Radio Astronomy Telescope. MEng thesis, Stellenbosch Univ. (2017).

\section{Acknowledgements}

The authors are grateful to the various organizations and funding bodies that have contributed immensely to the success of the Ghana Radio Astronomy Observatory. These include the Leverhulme-Royal Society Africa Awards, the Newton Fund via the DARA project, executed through the University of Leeds, UK, the South African Radio Astronomy Observatory, the IAU/OAD office in Cape Town and the governments of Ghana and South Africa. The authors are grateful to M.G. Hoare, Director of the DARA project, for his immense contribution to astronomy development in Ghana and Africa. E.P.-A. and T.A.-N. are also grateful to B. Abanga, H. Ayuk, C. Rhoe Quaye, F. Madjitey, J. Nanewortor, J. Allotey, D. Osei Owusu, R. Adonteng Asiamah, D. Sackey, E. Agbanu, E. Kofi Bosompem and the members of GSSTI and PRAGSAC who helped in various ways in reaching out to people with the message of astronomy.

\section{Competing interests}

The authors declare no competing interests. 